\title{
Simulações de Hinge Model utilizando Previsão Temporal da Evolução do DGD
}

\author{
J. Ferreira e J. P. von der Weid
}

\begin{abstract}
Resumo-Recentemente, foi proposto um novo modelo para análise estatística da PMD em sistemas de transmissão óptica, chamado de hinge model. A modelagem correta de um sistema está relacionada com a capacidade de análise de falhas e de correção de eventuais problemas, como PMD polarization mode dispersion. Nesse artigo é apresentado um estudo do hinge model, através de simulações computacionais baseadas num método de previsão do comportamento temporal do DGD (differencial group delay) em sistemas de transmissão a fibras ópticas. A capacidade de previsão de situações de falha desse método temporal é discutida.
\end{abstract}

Palavras-Chave-Polarisation mode dispersion (PMD), hinge model, evolução temporal, probabilidade de falha.

Abstract-Recently, a "hinge model" for optical fiber transmission link has been proposed. Modelling of the DGD temporal changes represents a critical aspect of outage probability characterization and PMD mitigation capacity. In this work some simulations of hinge model are presented considering a temporal evolution simulator. Outage probability using temporal simulations are discussed.

Keywords-Polarisation mode dispersion (PMD), hinge model, temporal evolution, outage probability.

\section{INTRODUÇÃO}

A PMD (Polarization Mode Dispersion) é considerada um dos grandes obstáculos para a realização dos sistemas com altas taxas de transmissão, $40 \mathrm{Gbit} / \mathrm{s}$ ou maiores. Este fenômeno possui natureza aleátoria, dependente da variação do ambiente e, portanto, gera grande incerteza no que concerne à sua compensação [5]. Simular fielmente as estatísticas e a dinâmica da PMD em sistemas reais de transmissão em fibras ópticas é um desafio no combate aos efeitos desse fenômeno [1].

$\mathrm{Na}$ modelagem usual dos sistemas ópticos que sofrem com o fenômeno da PMD, as amostragens temporais do DGD possuem a mesma distribuição maxwelliana para todas as frequências, significando que todos os canais são afetados pela PMD da mesma maneira [5]. Esta modelagem maxwelliana, é considerada clássica e se mostra excelente quando o DGD apresenta descorrelação rápida, para o domínio da frequência e do tempo [6]. Entretanto, apesar deste modelo ter sido aceito por um longo tempo, alguns trabalhos experimentais mostraram que nem sempre a estatística se apresenta exatamente dessa forma [7], [8], e mais recentemente [9]. Essa idéia, de que canais diferentes experimentam diferentes distribuições estatísticas, é a base de um modelo proposto por Brodsky et al. [10] e [11], chamado de hinge

J. Ferreira, Instituto Nacional de Metrologia, Normalização e Qualidade Industrial - Inmetro, J. P. Von der Weid, Centro de Estudos em Telecomunicações - PUC/RIO Emails: jferreira@inmetro.gov.br, vdweid@cetuc.puc-rio.br. model. Neste modelo, cabos subterrâneos são considerados como "congelados" para as variações rápidas, seguindo as variações sazonais lentas do ambiente. Por outro lado, pequenas distâncias de fibra, que se encontram expostas ao meio ambiente, ou elementos do sistema de transmissão localizados ao longo do enlace, como pontes, módulos de compensação e amplificação e salas de controle, agem como rotatores aleatórios de polarização conduzidos pelas variações de temperatura e vibrações mecânicas [12]. Estes rotatores aleatórios, chamados de hinges, são os responsáveis pelas variações temporais da PMD na escala de tempo rápida.

Um exemplo de aplicação desse modelo, foi publicado recentemente em [9]. Foram realizadas medidas em cabos ópticos enterrados, na cidade de Torino, na Itália, por 73 dias, para um sistema de múltiplos canais em uma faixa de $90 \mathrm{~nm}$. O DGD medido se mostrou bastante estável ao longo do tempo e para esse conjunto de dados obtidos a modelagem clássica maxwelliana parece ser inadequada, fazendo os autores suporem que o melhor ajuste seria pelo hinge model.

Os trabalhos mais recentes sobre esse tema apontam para um novo interesse: a análise de probabilidade de falhas dos sistemas de transmissão em face dessa nova modelagem [4] e [3]. Como os comprimentos de onda possuem estatística de PMD diferente entre si, esse comportamento acaba sendo refletido no comportamento da probabilidade de falha para diferentes canais. Os resultados dependerão do número de hinges e torna-se necessário a introdução de um novo conceito, chamado NCR (noncompliant capacity ratio), que está relacionado com a fração de comprimentos de onda que apresentam uma performance superior do que o esperado para uma determinada especificação de falha, num sistema de múltiplos comprimentos de onda.

Neste trabalho, na seção seguinte, apresenta-se a modelagem do simulador temporal, que permite previsão contínua ao longo do tempo. Na seção III são mostrados os resultados encontrados. As simulações computacionais de hinge model, neste trabalho, foram realizadas conectando-se em série, "fibras congeladas" (com variação muito lenta do DGD) com controladores de polarização ou fibras de menor valor de PMD. Nessas fibras de menor PMD, assim também como nos controladores de polarização, a variação do DGD acontece de forma mais veloz que nas "fibras congeladas", simulando assim, o efeito dos hinges. Finalmente, apresenta-se as conclusões.

\section{Modelagem do Simulador Temporal}

O fenômeno da PMD em fibras ópticas ocorre devido à birrefringência residual existente na fibra. Essa birefringência 
varia aleatoriamente ao longo do comprimento da fibra. As fibras são comumente modeladas como uma sequência de seções de birrefringência aleatória, cuja magnitude varia ao longo do comprimento da fibra [13].

Para realizar as simulações, neste trabalho, foi utilizado o formalismo de Jones. Através desse recurso matemático é calculada a matriz de tranferência da fibra. A matriz de Jones, $T(w)$, que descreve a fibra na frequência óptica $w$ é calculada por $N$ produtos sucessivos de duas matrizes [14]:

$$
T(w)=\prod_{n=1}^{N} B_{n}(w) R_{n}(\alpha)
$$

onde $B_{n}(w, t)$ representa a matriz de birrefringência do $\mathbf{n}$ ésimo segmento de fibra e $R_{n}(\alpha)$ é a matriz do rotator, representada pelo acoplamento aleatório em cada emenda.

Para realizar simulações da evolução temporal da fibra é introduzida uma função de variação aleatória da birrefringência da fibra. Logo, a equação 1 passa a ter uma variável temporal além da variável de frequência, $T(w, t)$ [15].

$$
T(w, t)=\prod_{n=1}^{N}\left(\begin{array}{cc}
e^{j \varphi / 2} & 0 \\
0 & e^{-j \varphi / 2}
\end{array}\right)\left(\begin{array}{cc}
\cos \alpha_{n} & \sin \alpha_{n} \\
-\sin \alpha_{n} & \cos \alpha_{n}
\end{array}\right)
$$

onde tem-se

$$
\varphi=\left[b_{n}(t) w h_{n}\right]
$$

onde $w$ é a frequência óptica, $b_{n}$ e $h_{n}$ representam o comprimento e a birrefringência de cada um dos segmentos de fibra e $\alpha_{n}$ é o ângulo de acoplamento entre estes segmentos.

Para a equação acima, a cada passo da simulação, representando a evolução temporal, a birrefringência de cada pedaço sofre uma variação determinada, linear a cada passo, mas a uma taxa diferente entre os pedaços. Após um certo número de iterações, um novo conjunto randômico para os passos $\delta_{n}$ é escolhido. A equação 4 mostra como esta variação foi inserida no simulador. Vale ressaltar que esse conjunto, $\delta_{n}$, possui valores positivos e negativos, de forma que a variação da birrefringencia seja limitada e tenha média igual a zero, para uma sequência infinita.

$$
b_{n}(t)=b_{n}(t-1)+\delta_{n} \cdot t
$$

\section{REsUltados}

A princípio decidiu-se testar qual seria a melhor forma de se representar o sistema de transmissão, pois é desejável que a variação do PSP, (Principal State of Polarization Estados Principais de Polarização), provocada pelos hinges seja maior que as variações devido ao comportamento das "fibras congeladas". Para tanto, foram consideradas duas possibilidades: o uso de misturadores de polarização (scramblers de polarização) e de fibras atuando como hinges. Primeiramente foram conectadas em série, fibras de $1 \mathrm{ps}$, que apresentavam variação lenta do DGD, com fibras de 1 ps com variação rápida. As fibras simuladas possuem $100 \mathrm{~km}$ de comprimento, cada. Num segundo momento, as mesmas fibras de $1 \mathrm{ps}$ com variação lenta do DGD foram conectadas a misturadores de polarização. A PMD das fibras "congeladas" variou lentamente, enquanto os hinges provocaram variações rápidas, cobrindo toda a esfera de Poincaré. A Figura 1 mostra uma comparação dos resultados obtidos. Foi simulado um sistema com 4 hinges, composto de 5 fibras de 1 ps e, entre elas, 4 misturadores de polarização de 3 lâminas ou 4 fibras de 1 ps. Ambos os casos mostram um comportamento parecido, havendo entretanto, um "deslocamento vertical da curva", no caso da utilização das 4 fibras de 1 ps. Essa variação entre as duas curvas, ou seja, uma diferença no valor do DGD médio, é devido a PMD adicional que é inserida para o caso que utiliza fibras no lugar dos misturadores de polarização.

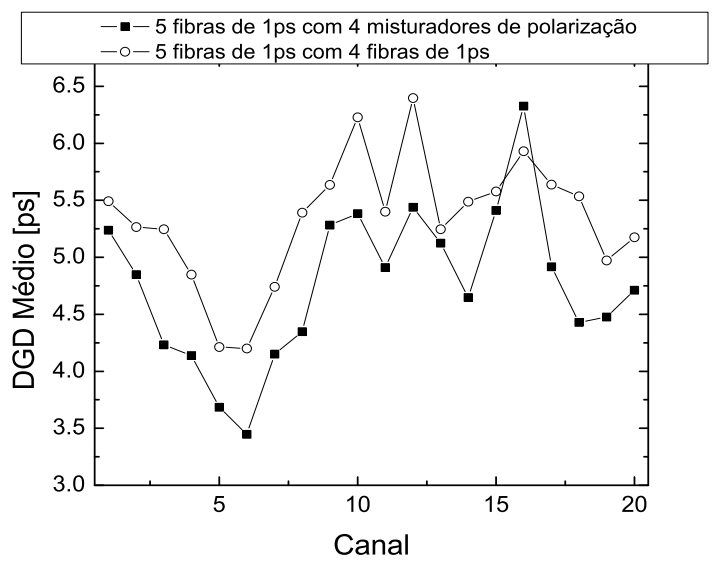

Fig. 1. Variação do DGD médio para diferentes canais WDM para um sistema com piezos e fibras no lugar dos hinges.

Como o resultado foi bastante parecido para os dois casos, foi escolhido trabalhar com os misturadores de polarização, devido a facilidade computacional da pré-geração de um volume significativo de dados.

Na Figura 2, é mostrado o DGD médio de vários canais ao longo do tempo. A pdf (probability density function - função densidade probabilidade) dos DGD's para cada canal é muito diferente da curva maxwelliana, devido ao pequeno número de hinges, mas a média do DGD tem uma variação muito próxima ao mostrado em [6] e [11].

É interessante mostrar que, conforme publicado em [2], alguns valores de DGD chegam a ser o dobro de outros canais. No trabalho citado, os dados são obtidos através de medidas. Na Tabela I, é mostrado um resultado obtido através de simulações temporais, onde o DGD médio para 20 canais varia em até $100 \%$.

No hinge model, cada canal vê uma distribuição diferente para o DGD (determinada principalmente pela escala de tempo rápida dos hinges). É possível, então, plotar o valor médio do DGD e seu desvio padrão para os canais, conforme mostrado na Figura 3. Nesta figura, foi realizada uma média ao longo dos 20 canais simulados, obtendo assim uma média espectral ao longo do tempo. O espalhamento da média do DGD varia de acordo com o tempo, logo, os canais terão um comportamento 


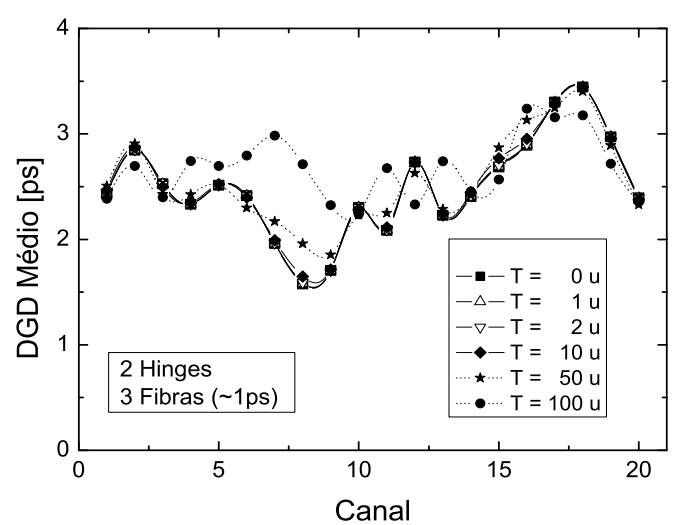

Fig. 2. Variação do DGD médio para diferentes canais WDM para uma transmissão em um sistema contendo 2 hinges e 3 fibras de 1 ps de PMD cada.

de correlação diferente, dependendo se a distribuição é mais ou menos larga. $\mathrm{O}$ espalhamento médio do DGD se torna menor à medida que o número de hinges aumenta, resultado semelhante ao apresentado em [10].

Uma abordagem que pode ser considerada, utilizando os dados de hinge model gerados com o simulador temporal, é o estudo de falha de sistema, ou seja, quando o nível de sinal fica abaixo de um determinado valor e a transmissão simplesmente é interrompida devido a erros que inutilizam o sinal. É possível especular sobre quanto tempo o sistema permanece em situações de falha, uma vez que ele tenha entrado nesta situação. Na Figura 4, é mostrada a probabilidade da duração de eventos de falha juntamente com a evolução temporal do DGD correspondente ao evento mais longo. Sendo $\langle\tau\rangle$ o valor médio do DGD, a probabilidade de que estes eventos ruins aconteçam, cai de $10^{-4}$ para $10^{-5}$ quando o nível máximo, aceito para o DGD, varia de $2<\tau>$ para $2,5<\tau>$ e mais de uma ordem de magnitude quando varia para $3<\tau>$. Mesmo assim, a duração do evento é considerada alta $(\sim 40 u)$ mesmo para o caso com maior DGD.

\begin{tabular}{r||c|c|c|c|c}
\hline \hline Canal & 1 & 2 & 3 & 4 & 5 \\
\hline DGD médio [ps] & 4,17 & 4,83 & 3,60 & 3,00 & 3,33 \\
\hline \hline Canal & 6 & 7 & 8 & 9 & 10 \\
\hline DGD médio [ps] & 3,92 & 3,86 & 3,96 & 3,22 & 2,49 \\
\hline \hline Canal & 11 & 12 & 13 & 14 & 15 \\
\hline DGD médio [ps] & 4,03 & 3,79 & 4,12 & 3,40 & 3,23 \\
\hline \hline Canal & 16 & 17 & 18 & 19 & 20 \\
\hline DGD médio [ps] & 4,03 & 3,79 & 4,12 & 3,40 & 3,23 \\
\hline
\end{tabular}

TABELA I

DGD MÉDIO DE 20 CANAIS UTILIZANDO MODELAGEM hinge model.

Utilizando as simulações no domínio do tempo, é possível ajustar a escala de tempo desses resultados com a escala de tempo de flutuações observadas numa fibra instalada e determinar a estatística da duração de eventos de falha, através

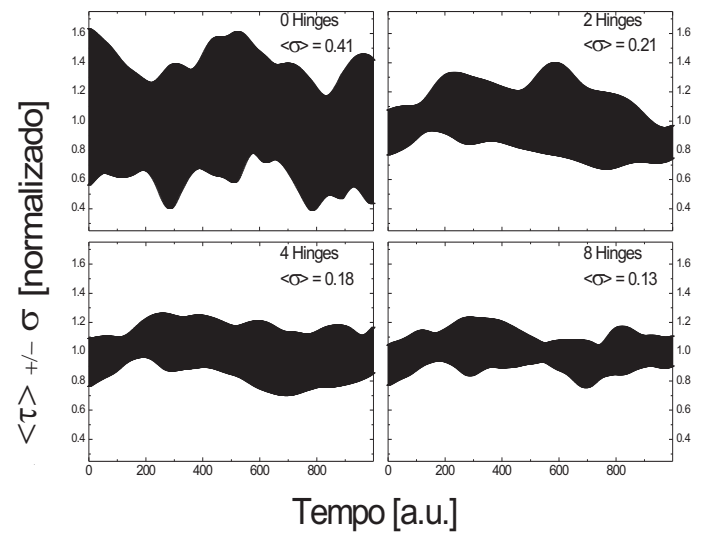

Fig. 3. Evolução temporal do DGD médio e seu desvio padrão para diferentes números de hinges.

de comparações com medidas já realizadas. Isto é possível através da associação entre os dados gerados nesse trabalho com alguma unidade real de medida temporal (horas, minutos ou segundos), a partir de dados medidos da evolução da PMD ao longo do tempo. Para exemplificar essa correspodência, foi escolhidos um trabalho, [16], que reporta medidas de PMD em função do tempo com o valor da PMD em torno de 2 ps, mesmo valor gerado nas simulações. Nesse caso, cada ponto simulado corresponde a $0,22 \mathrm{~h}$, e portanto é possível prever quanto tempo o sistema permaneceria em uma situação de falha.

\section{Conclusões}

Foi discutido um modelo para a PMD, o hinge model, prosposto recentemente em [11] e [10]. As variações lentas na evolução da PMD nos sistemas quando os cabos estão enterrados acontecem devido às variações sazonais nas condições do ambiente. Neste modelo é assumido que fibras que apresentam essa variação lenta estão intercaladas com elementos (pontes, por exemplo) que produzem variações rápidas no valor do DGD, ou seja, que agem como rotatores de polarização [9]. E estas variações se demonstram de forma diferente no caso de transmissão de múltiplos canais. Neste caso, os canais mostram distribuições estatísticas diferentes, conflitando com o modelo clássico onde todos os canais possuem a mesma distribuição maxwelliana.

Os resultados encontrados, para as simulações de hinge model estiveram de acordo com as expectativas, sendo percebida uma variação de aproximadamente $100 \%$ entre o DGD médio de 20 canais, para um sistema composto por dois hinges e três fibras de $\sim 1$ ps. Também foi visto que o número de hinges do sistema exerce uma forte influência na estatística dos canais, sendo que quanto maior o número de hinges menor é o espalhamento do valor do DGD entre os canais.

A estatística de duração dos eventos de falha (DGD alto e baixa potência do sinal de RF no receptor) pode ser determinada utilizando-se o modelo temporal apresentado, agregando informações da modelagem de hinge model e de aplicações 


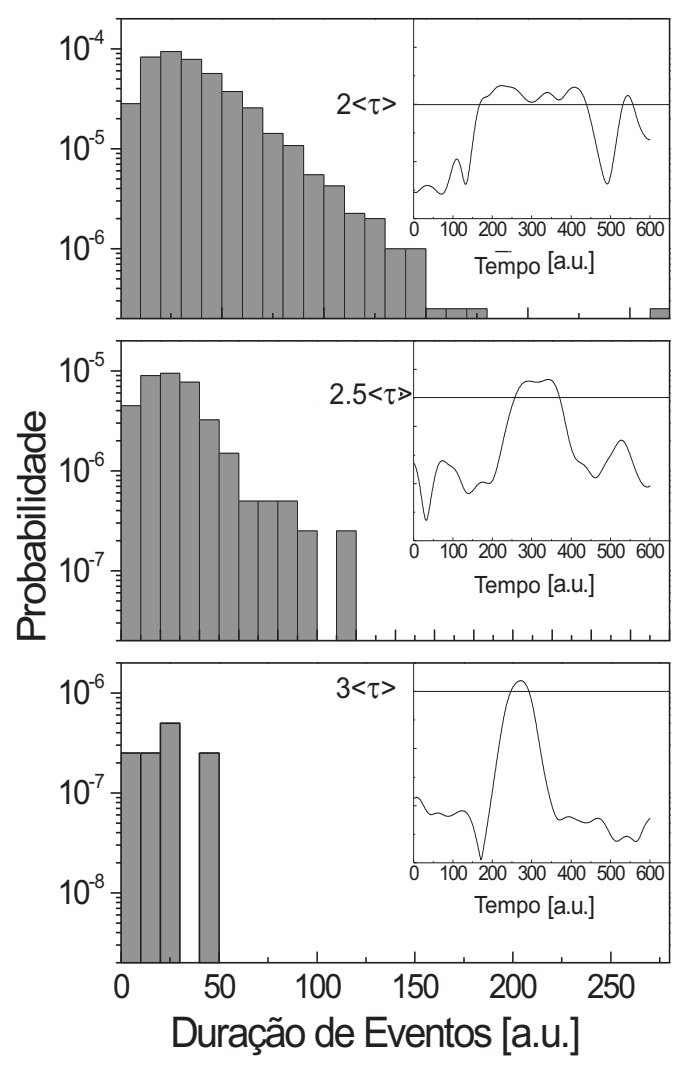

Fig. 4. Histograma de eventos de falha para diferentes valores de DGD. O detalhe mostra a evolução temporal do pior evento para cada um dos valores de DGD médio.

práticas que necessitam de informações contínuas da situação instantânea do sistema.

\section{REFERÊNCIAS}

[1] L.-S. Yan and X. S. Yao and A. E. Willner, Enabling "hinge" model in polarization-mode-dispersion statistics using variable differential-groupdelay-based emulator, IEEE Photonics Technology Letters, v. 18, n. 2, pp. 427-429, January, 2006.

[2] , M. Brodsky and M. Boroditsky and P. Magill and N. J. Frigo, Persistence of spectral variations in DGD statistics, Optics Express, v. 13, n. 11, pp. 4090-4095, May, 2005.

[3] H. Kogelnik and J. Winzer, PMD outage probabilities revisited, Optical Fiber Communication and the National Fiber Optic Engineers Conference, 2007. OFC/NFOEC 2007. Conference on e, paperOTuN3, March, 2007.

[4] X. Chongjin, L. Xiang and H. Bulow, P PMD-Induced Outage Probability in an Optical Communication System With Fast Polarization Scramblers and Time-Varying PMD Sections, Photonics Technology Letters, v. 20, n. 6, pp. 440-442, March, 2008.

[5] C. Antonelli and Antonio Mecozzi, Theoretical characterization and system impact of the hinge model of PMD. Journal of Lightwave Technology, v. 24, n.11, pp. 4064-4074, November, 2006.

[6] M. Karlsson, J. Brentel, and A. Andrekson, Long-term measurement of $P M D$ and polarization drift in installed fibers, Journal of Lightwave Technology, v. 18, n.7, pp. 941-951, July, 2000.

[7] R. Caponi and B. Riposati and A. Rossano and M. Schiano, WDM design issues with highly correlates WDM spectra of buried optical cables. Optical fiber Communication, paper ThI5, 2002.

[8] M. Schiano, PMD measurements on installed fibers and polarization sensitive components, PMD Venice Summer School, 2002.
[9] P. Poggiolini and A. Nespola and S. Abrate and V. Ferrero and C. Lezzi, Long-term PMD characterization of a metropolitan G.652 fiber plant, Journal of Lightwave Technology, v. 24, n. 11, pp. 4022-4029, November, 2006.

[10] M. Brodsky, M. Boroditsky, P. Magill, N. J. Frigo and M. Tur, Channelto-channel variation of non-maxwellian statistics of DGD in a field installed system, Eur. Conf. Optical Communication - ECOC 2004, v. 3, pp. 306-309, 2004.

[11] M. Brodsky, M. Boroditsky, P. Magill, N. J. Frigo and M. Tur, A "hinge" model for the temporal dynamics of polarization mode dispersion, Proc. 17th Annu. Meeting IEEE Laser and Electro-Optics Society (LEOS 2004), v. 1, n. 7-11, pp. 90-91, November, 2004.

[12] M. Brodsky and M. Boroditsky and N. J. Frigo and P. Magill and M. Tur, Field PMD measurements through a commercial Raman-amplified ULH transmission system, LEOS PMD Summer Topical Meeting, paper MB3.3, 2003.

[13] J. P. Gordon and H. Kogelnik, PMD fundamentals: polarization mode dispersion in optical fibers, Proceedings of the National Academy of Sciences of the USA, v. 9, n. 97, pp. 4541-4550, April, 2000.

[14] A. A.O. dal Forno, R. P. A. Paradisi and J. P. von der Weid, Experimental and theoretical modeling of polarization-mode dispersion in single fibers, IEEE Photonics Technology Letters, v. 3, n. 12, pp. 296-298, March, 2000.

[15] J. Ferreira and J. P. von der Weid, Time domain PMD simulation in optical fibers and emulators, IEEE/LEOS Workshop of Fibers and Optical Passive Components, pp. 176-180, June, 2005.

[16] A. C. Antonelli, M. B. A. Mecozzi and M. Boroditsky, A simple analytical model for PMD temporal evolution, Optical Fiber Communication Conference OFC'2006, March, 2006. 